\title{
CD176 antiserum treatment leads to a therapeutic response in a murine model of leukemia
}

\author{
BIN YI $^{1,2^{*}}$, ZHE ZHANG $^{1,2^{*}}$, MIN ZHANG $^{1,2}$, REINHARD SCHWARTZ-ALBIEZ $^{3}$ and YI CAO $^{1}$ \\ ${ }^{1}$ Laboratory of Molecular and Experimental Pathology, Kunming Institute of Zoology, Chinese Academy of Sciences, \\ Kunming, Yunnan; ${ }^{2}$ Graduate University of the Chinese Academy of Sciences, Beijing, P.R. China; \\ ${ }^{3}$ Translational Immunology Unit, German Cancer Research Center, Heidelberg, Germany
}

Received April 28, 2013; Accepted June 21, 2013

DOI: $10.3892 /$ or.2013.2639

\begin{abstract}
CD176 (Thomsen-Friedenreich antigen) is a tumorassociated carbohydrate structure. CD176 is expressed at the surface of human leukemic cells but is almost absent in normal and benign adult human tissues. Therefore, CD176 could be a promising target for antitumor immunotherapy. In the present study, pre-immunization with asialoglycophorin A (containing the CD176 oligosaccharide chains) was able to significantly improve the survival time of mice carrying $\mathrm{CD}_{176}{ }^{+}$leukemia as compared to the control mice without the immunization. Furthermore, the passive transfer of CD176 antiserum which reacted only with the tumor-associated CD176 in cancer cells, was able to effectively prolong the survival time of $\mathrm{CD} 176^{+}$ leukemia mice. In particular, the CD176 antiserum treatment could inhibit the growth and spreading of $\mathrm{CD}_{176^{+}}$leukemic cells in bone marrow, spleen, liver, and lung as evidenced by histopathological examination. CD176 antiserum could induce the apoptosis of $\mathrm{CD}_{176^{+}}$leukemic cells in vivo in a manner as previously observed in vitro. The data provided strong evidence that both CD176 antigen-based active immunotherapy and CD176 antibody-based passive immunotherapy
\end{abstract}

Correspondence to: Professor Yi Cao, Laboratory of Molecular and Experimental Pathology, Kunming Institute of Zoology, Chinese Academy of Sciences, 32 Jiaochang Donglu, Kunming, Yunnan 650223, P.R. China

E-mail: caoy@mail.kiz.ac.cn

*Contributed equally

Abbreviations: TF, Thomsen-Friedenreich antigen; DTH, delayed type hypersensitivity; aGP, asialoglycophorin; mAb, monoclonal antibody; PAA, polyacrylamide; PBS, phosphate-buffered saline; VCN, vibrio cholerae neuraminidase (sialidase); ELISA, solid-phase enzyme linked immunosorbent assay; BSA, bovine serum albumin; PI, propidium iodide; H\&E, hematoxylin and eosin; ssDNA, single stranded DNA; ALL, acute lymphoblastic leukemia; CDC, complement-dependent cytotoxicity; ADCC, antibody-dependent cellular cytotoxicity

Key words: CD176, Thomsen-Friedenreich antigen, antibody, leukemia, immunotherapy, mouse lead to a therapeutic response in $\mathrm{CD} 176^{+}$leukemia mice. Therefore, both CD176 vaccine and CD176 antibody drug may be beneficial for the treatment of $\mathrm{CD} 176^{+}$leukemia patients.

\section{Introduction}

Thomsen-Friedenreich (TF) antigen is a tumor-associated carbohydrate structure which is defined as the carbohydrate epitope (glycotope) sequence Gal $\beta 1-3$ GalNAc $\alpha 1-R$ (1). TF was assigned as CD176 during the 7th Conference on Human Leucocyte Differentiation Antigens (2). In adult human normal and benign tissues, CD176 is masked by terminal sialylation (3), but it is exposed during tumorigenesis as a tumor-associated antigen (4). Approximately 70-80\% of carcinomas carry CD176 on their cell surface (5). CD176 is also expressed in leukemia and lymphoma cells $(4,6)$, and may be a promising prognostic marker in T-cell acute lymphoblastic leukemia $(7,8)$. In addition, CD176 is expressed in some cancer stem cells (9), and it is functionally involved in the liver metastasis process of tumors $(1,10)$, the adhesion of human breast cancer cells to the endothelium $(11,12)$ and the apoptosis of leukemic cells (13). Therefore, CD176 may be a promising target for cancer immunotherapy.

Immunotherapy using a CD176 vaccine has been studied on patients with advanced breast carcinoma (4,5), ovarian carcinoma (14) and prostate carcinoma (15). These studies demonstrated that the CD176 (TF) vaccine was effective and safe for the patients. In the patients immunized with CD176 antigen, CD176-specific delayed type hypersensitivity (DTH; 4,5) and high-titer CD176 IgM and IgG antibodies (15) were observed, indicating that both responses of cellular and humoral immunity are involved in this immunotherapeutic approach.

Antitumor antibodies applied in cancer immunotherapy are effective for a variety of hematological malignancies (16). In previous studies, we observed that CD176 antibody can induced apoptosis of CD176 ${ }^{+}$leukemic cells through activation of the CD95 pathway and caspase-3 $(13,17)$. Liver colonization of CD176 (TF)-positive syngeneic tumor cells was suppressed by pretreatment with a CD176 (TF) antibody (18). CD176 (TF) antibody markedly inhibited the adhesion of human breast cancer cells to the endothelium and caused an extension of survival time in mice carrying a 4T1 metastatic breast 
tumor (12). Based on these observations, we hypothesized that CD176 antibody might have a therapeutic effect on patients with $\mathrm{CD}_{176^{+}}$cancer. In the present study, we investigated: i) whether the therapeutic effect could be observed by immunization with the CD176 antigen; and ii) whether treatment with the CD176 antibody treatment alone leads to a therapeutic response in mice inoculated with $\mathrm{CD}_{176^{+}}$leukemic cells. The present study aims to open new strategies for the development of a CD176 antibody drug.

\section{Materials and methods}

Mice and cell lines. Eight to ten-week-old BALB/c mice (Experimental Animal Center, Kunming Medical College, China) were housed at the Animal Facility, Kunming Institute of Zoology, Chinese Academy of Science. Both male and female mice were used. All experiments were performed in accordance with the regulation for animal experimentation and approved by the responsible authorities.

Cell lines of WEHI-3 (murine myelomonocytic leukemia) and KG-1 (human acute myelogenous leukemia) were purchased from the American Type Culture Collection (ATCC, Manassas, VA, USA). WEHI-3 and KG-1 cells were cultured in DMEM and RPMI-1640 medium (Gibco-Invitrogen, Grand Island, NY, USA), supplemented with 10 and $20 \%$ fetal bovine serum, respectively, and penicillin and streptomycin. For the generation of CD176 ${ }^{+}$WEHI-3, WEHI-3 cells were pretreated with VCN (sialidase from Vibrio cholerae; Sigma, St. Louis, MO, USA) for $1 \mathrm{~h}$ to remove NeuAc in 2-3, 2-6 and 2-8 linkages.

Reagents and antibodies. Asialoglycophorin $\mathrm{A}(\mathrm{aGP})$ and TF (CD176)-polyacrylamide (TF-PAA) were purchased from International Laboratory Ltd., (San Francisco, CA, USA) and Glyco Tech Corp. (Gaithersburg, MD, USA), respectively.

CD176 antiserum was generated by subcutaneously injecting mice with $40 \mu \mathrm{g}$ aGP together with incomplete Freund's adjuvant (Sigma). Following two-boosting immunization every two weeks, $100 \mu \mathrm{l}$ of blood were collected by tail bleeding. An indirect solid-phase enzyme linked immunosorbent assay (ELISA) was used as described below to assess the titer of CD176 antibody in the sera. The specific reaction of the CD176 antiserum was determined by inhibition experiments using the inhibitor TF-PAA as described below. When titers of the CD176-specific antibodies of IgM and IgG isotype reached 1:1,000, the sera were collected and filtered.

ELISA assay. TF-PAA or aGP were coated on microplates, and then blocked with $2 \%$ bovine serum albumin (BSA). Following incubation with sera from the immunized mice, the wells were treated with peroxidase-labeled goat anti-mouse $\operatorname{IgM}$ ( $\mu$-chain specific; Southern Biotech, Birmingham, AL, USA) or peroxidase-labeled goat anti-mouse $\operatorname{IgG}(\mathrm{H}+\mathrm{L})$. The color reaction was developed with a solution of o-phenylenediamine dihydrochloride. Negative controls were performed using the normal mouse serum instead of the CD176 antiserum.

Inhibition experiments based on ELISA, cell-ELISA and immunofluorescence staining. The WEHI-3 cells were treated with VCN before harvesting. The cells were lysed with cell lysis buffer and centrifuged. The supernatants were coated on microplates, and then blocked with $2 \%$ BSA. Following incubation with the CD176 antiserum or the CD176 antiserum plus TF-PAA, the wells were supplemented with peroxidase-labeled goat anti-mouse immunoglobulin. The color development was previously described.

For cell-ELISA, WEHI-3 cells were cultured in the 96-well plate for 2 days. The cells were treated with VCN, and were then fixed with $4 \%$ paraformaldehyde. After blocking with $2 \%$ BSA, the cells were incubated with the CD176 antiserum or the CD176 antiserum plus TF-PAA. Subsequently, the cells were treated with peroxidase-labeled goat anti-mouse immunoglobulin. The color reaction was previously described.

For immunofluorescence staining, WEHI-3 cells grown on cover slips coated with poly-L-lysine were treated with VCN and then fixed in acetone. After blocking with $1 \%$ BSA, the cells were treated with the CD176 antiserum or the CD176 antiserum plus TF-PAA. Then, the cells were incubated with FITC-labeled goat anti-mouse immunoglobulin and counterstained with DAPI (Beyotime Biotechnology Co., Ltd., Shanghai, China). The cover slips were mounted with glycerol and the images were viewed and captured.

Induction of apoptosis with CD176 antiserum in vitro. WEHI-3 cells were treated with VCN, and were then incubated with the CD176 antiserum for 22 and $26 \mathrm{~h}$, respectively. Apoptotic cells were assessed by using the Annexin V-FITC Apoptosis Detection kit (KGA107; KeyGen, Nanjing, China) and propidium iodide (PI) according to the manufacturer's instructions using a FACScan flow cytometer (BD Biosciences, Rockville, MD, USA). WEHI-3 cells were treated with VCN and normal mouse serum instead of the CD176 antiserum as negative controls. KG-1 was used as positive control (13).

Initiative immunotherapy using the CD176 antigen. In initiative immunotherapy experiments, we investigated the effect of aGP immunization on the survival time of $\mathrm{CD} 176^{+}$leukemia mice. Forty mice were randomly divided into 2 groups ( 20 mice per group). The first group was immunized subcutaneously at flanks and base of backs with $40 \mu \mathrm{g}$ aGP together with incomplete Freund's adjuvant (Sigma) for three times. ELISA was used to estimate titers of the CD176 antibody in the sera. When the antibody titers reached 1:1,000, the mice were intravenously

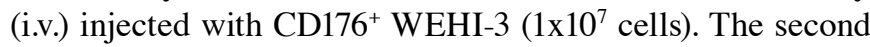
group was only i.v. injected with CD176 ${ }^{+}$WEHI- $3\left(1 \times 10^{7}\right.$ cells $)$ as a control. Mice were weighed and observed on a daily basis. The survival time was recorded and analyzed.

Passive immunotherapy using the CD176 antiserum. In the first experiments of antibody treatment, we studied effects of the CD176 antibody treatment on the survival time of $\mathrm{CD}_{176}{ }^{+}$leukemia mice using passive transfer of the CD176 antiserum. Sixty mice were randomly divided into 3 groups with twenty mice in each group. Group 1 was injected i.v. with $1 \times 10^{7} \mathrm{CD}_{176^{+}} \mathrm{WEHI}-3$ cells; group 2 was injected i.v. with $1 \times 10^{7} \mathrm{CD} 176^{+} \mathrm{WEHI}-3$ cells, and then treated i.v. with normal mouse serum three times (2nd day/700 $\mu 1$, 5th day/600 $\mu 1$ and 9th day $/ 500 \mu \mathrm{l}$ ) after cell injection. Group 3 was injected i.v. with $1 \times 10^{7} \mathrm{CD}_{176^{+}}$WEHI-3 cells, and then treated i.v. with the CD176 antiserum three times (2nd day/700 $\mu 1$, 5th day/600 $\mu 1$ and 9th day $/ 500 \mu \mathrm{l}$ ) after cell injection. The analysis of 
specificity and titer determination of CD176 antibody in the sera were previously described. The survival was monitored daily by two investigators. Clinical symptoms, such as rapid and labored breathing, paralyzed hind limbs and rough coat were recorded and used as indicators of morbidity. Mice that survived for more than 65 days were sacrificed.

In the second set of experiments of antibody treatment, we studied effects of the CD176 antibody treatment on the growth and spreading of $\mathrm{CD}_{176^{+}}$cancer cells in vivo. Eighty mice were used (20 mice in each group). The design and method applied were identical for group 1,2 and 3 as mentioned above. Group 4 consisted of normal mice without treatments. All mice of the 4 groups were sacrificed on the 19th day. The organs, such as lung, liver, spleen and kidney, were isolated, weighed, observed and photographed. The number of the liver and lung cancer spreading nodules in each group was counted. The tissues and bone marrow samples were used for further histopathological analysis.

Histopathology and immunohistochemistry. All mice (control and experimental groups) were weighed before being sacrificed. Liver, spleen, lung and kidney from each group were weighed, observed and photographed, and then fixed in the $4 \%$ formaldehyde and embedded in the paraffin. Paraffin sections were stained with hematoxylin and eosin (H\&E).

For analysis of apoptotic cells in tissue sections using antisingle stranded DNA (ssDNA) monoclonal antibody (mAb), tissues were fixed in cold $4 \%$ paraformaldehyde and cut into sections. The slides were immersed in methanol. Subsequently, the slides were treated with $10 \mu \mathrm{g} / \mathrm{ml}$ proteinase $\mathrm{K}$ at $56^{\circ} \mathrm{C}$ and then with $50 \%$ formamide. The slides were then treated with $3 \% \mathrm{H}_{2} \mathrm{O}_{2}$ and blocked with $3 \%$ BSA. Anti-ssDNA mAb (Chemicon, Temecula, CA, USA) and peroxidase-labeled goat anti-mouse immunoglobulin were used as the primary and secondary antibody, respectively. Color was developed with the peroxidase substrate 3,3'-diaminobenzidine. Counterstaining was performed with hematoxylin. Negative controls were performed with an irrelevant $\operatorname{IgM}$ from a mouse plasmocytoma (Sigma) at a comparable dilution instead of the mAb. Tissue sections which were not treated with proteinase $\mathrm{K}$ and heated formamide were used for the detection of CD176. CD176 mAb (19) was applied as the first antibody.

Bone marrow smears. The bone marrow was washed out from the femur of sacrificed mice treated with the CD176 antiserum on the 19th day after the inoculation of CD176 ${ }^{+}$WEHI-3. The bone marrow smear was carried out by pushing two slides and then Giemsa staining was applied. Leukemia cell numbers were counted by microscopy. The proportion of leukemia cells to total bone marrow cells was estimated in ten optical fields.

Statistical analysis. Data (number of liver tumor metastasis nodules, swelling degree of liver and spleen, the proportion of leukemia cells to total bone marrow cells) were analyzed using the paired t-test. Incidences of liver and lung tumor metastasis were compared with the Fisher's exact test (two-tailed). The Kaplan-Meier curves were used to determine survival differences between the CD176 antiserum treatment group or CD176 antigen vaccination group and their control groups. Log-rank test was used to determine the differences between curves.
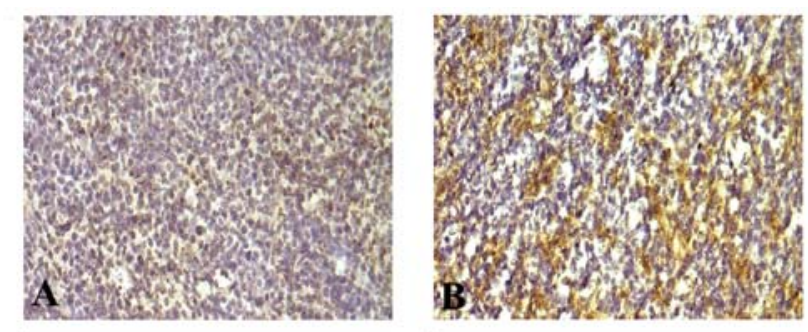

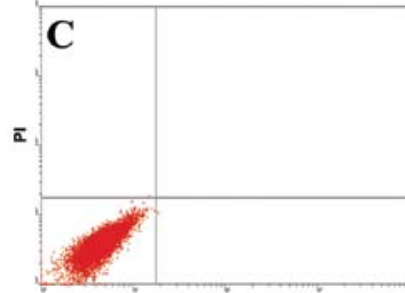

FITC-Annexin V

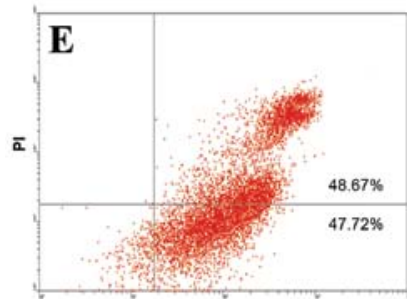

FITC-Annexin V
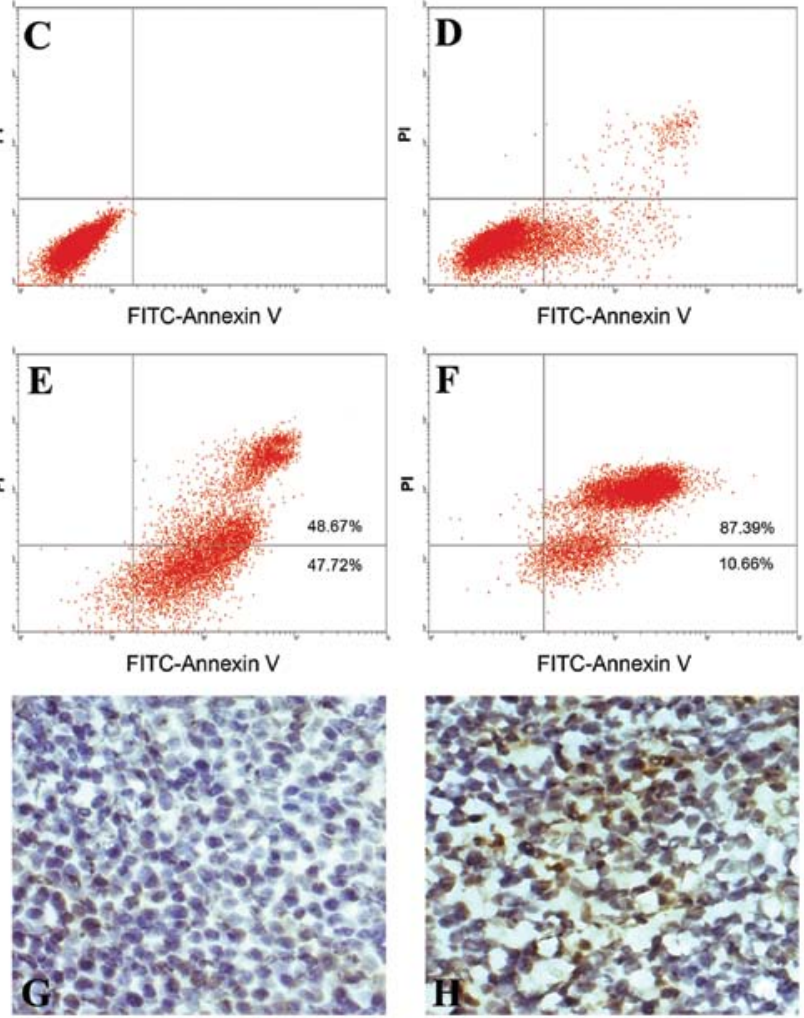

FITC-Annexin V

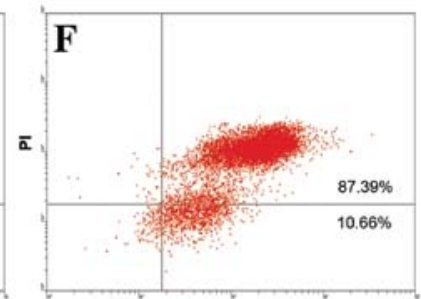

FITC-Annexin V

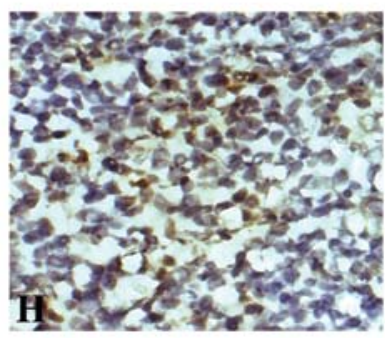

Figure 1. CD176 is strongly expressed in leukemic cells of liver metastasis nodules (A and B), and the CD176 antiserum induced apoptosis of CD176 WEHI-3 examined by Annexin V-FITC and PI staining and flow cytometry analysis (C-F) in vitro as well as immunohistological staining using the anti-ssDNA monoclonal antibody ( $\mathrm{G}$ and $\mathrm{H}$ ) in vivo. (A) Negative control using mouse IgM; (B) positive staining using CD176 monoclonal antibody (magnification, x200); (C) blank control; (D) treatment with normal mouse serum (1:10 for $22 \mathrm{~h}$ ); (E) treatment with the CD176 antiserum (1:10 for $22 \mathrm{~h}$ ); (F) treatment with the CD176 antiserum (1:10 for $26 \mathrm{~h}$ ). (G) Cancer cells in liver of the $\mathrm{CD}_{176^{+}}$leukemia mouse treated with the normal mouse serum; (H) cancer cells in liver of CD176 ${ }^{+}$leukemia mouse treated with the CD176 antibody (magnification, $\mathrm{x} 400$ ).

A value of $\mathrm{P}<0.05$ was considered to indicate a statistically significant result.

\section{Results}

Establishment of $\mathrm{CD}_{176^{+}}$leukemic transplanted mice models. The mice injected with $\mathrm{CD}_{176^{+}}$WEHI-3 were investigated histologically and immunohistologically. Under histopathological observation, large numbers of scattered cancer cells and cancerous nodules were observed in lung, spleen, liver and bone marrow from the mice injected i.v with CD176 ${ }^{+} \mathrm{WEHI}-3$ cells but not in those left untreated mice. CD176 was strongly expressed in the cancer cells as observed by immunohistological analysis (Fig. 1A and B). A mouse model of CD176 ${ }^{+}$ leukemia transplants was established successfully. 

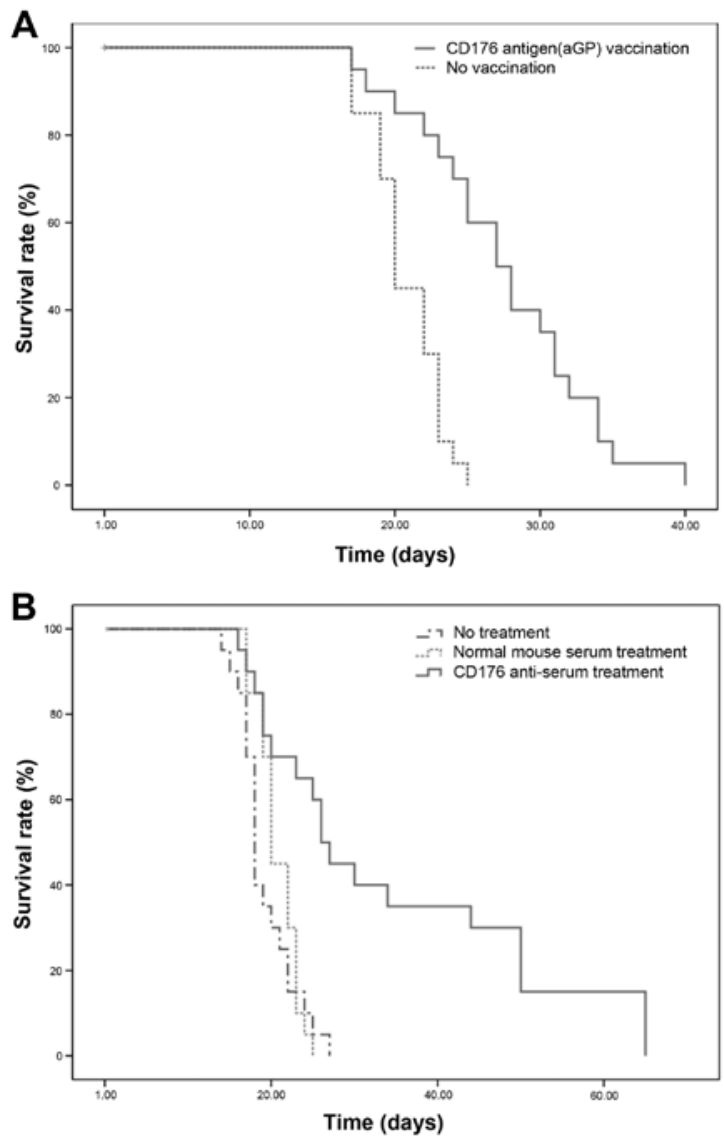

Figure 2. The survival time of $\mathrm{CD} 176^{+}$leukemic mice with the $\mathrm{CD} 176$ antigen (aGP) pre-vaccination and the CD176 antiserum treatment. (A) CD176 leukemic mice with aGP pre-vaccination had a significant survival advantage in comparison to the $\mathrm{CD}_{176^{+}}$leukemic mice without the pre-vaccination as assessed by the Kaplan-Meier analysis $(\mathrm{P}<0.001)$. (B) The CD176 antiserum treatment significantly prolonged the survival time of the $\mathrm{CD}_{176}$ leukemic mice (Kaplan-Meier analysis, $\mathrm{P}<0.001)$.

Apoptosis of $\mathrm{CD} 176^{+}$cancer cells induced by the CD176 antiserum treatment in vitro and in vivo. The CD176 antiserum induced apoptosis of $\mathrm{CD} 76^{+} \mathrm{WEHI}-3$ in vitro and in vivo by flow cytometry and histochemistry examination (Fig. 1C-H), indicating that the CD176 antiserum could kill CD176 ${ }^{+}$ leukemia cells by inducing the apoptosis of $\mathrm{CD} 176^{+}$leukemia cells.

Specific reaction of the CD176 antiserum with the tumorassociated CD176. The reaction of the CD176 antiserum with aGP was partially inhibited in ELISA using the inhibitor TF-PAA (data not shown). However, the reaction of the CD176 antiserum with the tumor-associated CD176 was almost completely inhibited in cell-ELISA and ELISA (data not shown). Furthermore, the staining of the CD176 antiserum at the surface of cancer cells was also clearly reduced using the inhibitor TF-PAA as seen in immunofluorescence staining (data not shown). Although the CD176 antiserum prepared from aGP also binds to peptides of aGP, the antibody binding peptides of aGP in the serum do not react with cancer cells, due to the absence of aGP peptides in cancer cells. These data demonstrated that the CD176 antiserum prepared from aGP reacted only with the tumor-associated CD176 in cancer cells.

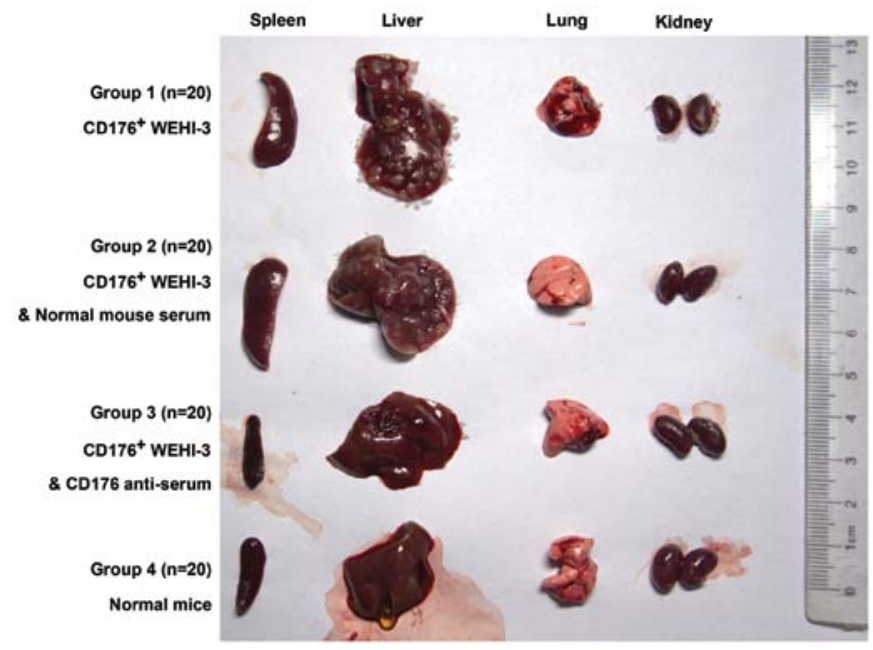

Figure 3. CD176 antiserum treatment affects the size and weight of the liver and spleen of $\mathrm{CD}_{176^{+}}$leukemic mice.
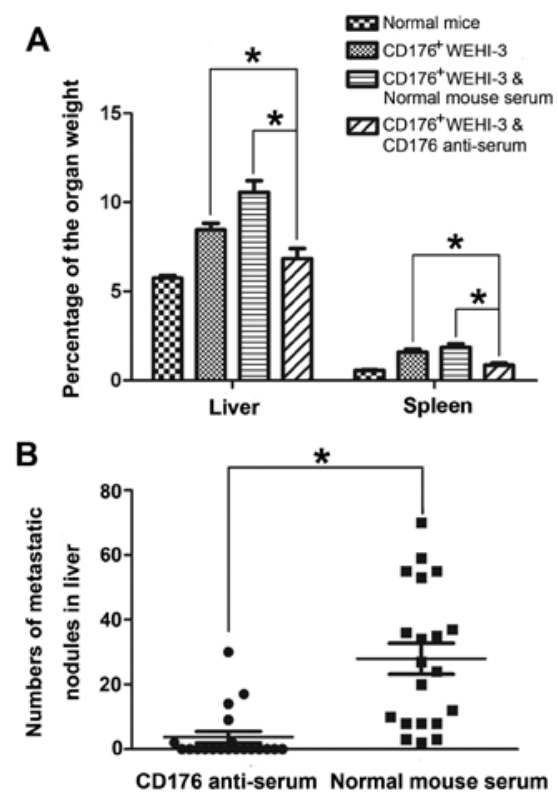

Figure 4. CD176 antiserum treatment reduces the degree of swelling of the liver and spleen and inhibits the metastasis of $\mathrm{CD}_{176}{ }^{+}$cancer cells in liver. (A) CD176 antiserum treatment significantly reduced the liver and spleen weight as compared to the total body weight (given as percentages, ${ }^{*} \mathrm{P}<0.001$ ). (B) The CD176 antiserum treatment significantly decreased the number of metastatic nodules in the liver $\left({ }^{*} \mathrm{P}<0.001\right)$.

The survival time of leukemic mice is prolonged by immunization using CD176 antigen. In initiative immunotherapy experiments, the leukemia mice pre-immunized with aGP $(n=20)$ had a significantly enhanced survival time in comparison to the non-immunized mice $(\mathrm{n}=20)$ as determined by the Kaplan-Meier analysis $(\mathrm{P}<0.001$; Fig. 2A). Compared with the control group, the median survival time of the immunized group was prolonged by 7 days and the survival time was increased by $32.8 \%$.

The survival time of the leukemic mice is prolonged by the CD176 antiserum treatment alone. In the first experiments of antibody treatment, the $\mathrm{CD}_{176^{+}}$leukemia mice that were 
Table I. Incidence of liver and lung metastasis between CD176 antiserum-treated mice and control mice.

Incidence of liver tumor metastasis $\mathrm{n}(\%)$
Incidence of lung tumor metastasis n $(\%)$

Groups

$\begin{array}{cl}16 / 20(80)^{\mathrm{a}} & 9 / 20(45)^{\mathrm{b}} \\ 20 / 20(100)^{\mathrm{a}} & 2 / 20(10) \\ 6 / 20(30)^{\mathrm{a}} & 0 / 20(0)^{\mathrm{b}}\end{array}$

${ }^{a}$ Number of relevant cases/total number of cases; $\mathrm{P}<0.001$. NMS, normal mouse serum. The CD176 antiserum treatment significantly reduced incidence of liver tumor metastasis compared to the two control groups, and decreased incidence of lung tumor metastasis compared to control mice injected with CD176 ${ }^{+}$WEHI-3 ( $\left.\mathrm{P}<0.001\right)$.

Table II. Percentage of leukemia cells in total bone marrow cells between CD176 antiserum-treated mice and control mice.

\begin{tabular}{lrrr}
\hline & \multicolumn{2}{c}{ Percentage of leukemia cells in total bone marrow cells } \\
\cline { 2 - 4 } Groups & $+^{\mathrm{a}}$ & ++ \\
\hline CD176 ${ }^{+}$WEHI-3 (n=6) & $0 / 6^{\mathrm{b}}$ & $0 / 6$ & $6 / 6$ \\
CD176 ${ }^{+}$WEHI-3 and NMS (n=6) & $0 / 6$ & $0 / 6$ & $6 / 6$ \\
CD176 ${ }^{+}$WEHI-3 and CD176 antiserum (n=6) & $5 / 6$ & $1 / 6$ & $0 / 6$
\end{tabular}

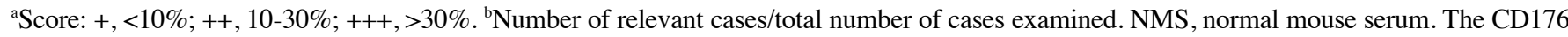
antiserum treatment significantly reduced the percentage of leukemic cells in total bone marrow cells compared to the two control groups $(\mathrm{P}<0.01)$.

treated with the CD176 antiserum alone after inoculation of CD176 ${ }^{+}$WEHI-3 ( $\left.n=20\right)$, showed a significant survival advantage in comparison to the mice treated with normal mouse serum $(n=20)$ and the mice without the treatment $(n=20)$ through the Kaplan-Meier analysis $(\mathrm{P}<0.001$; Fig. 2B). The median survival time of the CD176 antiserum treatment group was prolonged by 6 and 8 days compared with the two control groups, respectively.

The growth and spreading of $\mathrm{CD} 176^{+}$cancer cells is inhibited by the CD176 antiserum treatment alone. In the second set of antibody treatment experiments, several changes were observed in the leukemia mice treated with the CD176 antiserum $(n=20)$ as compared to the leukemia mice treated with normal mouse serum $(n=20)$ and without the treatment $(n=20)$ : i) swelling degrees of liver and spleen were significantly reduced $(\mathrm{P}<0.001$; Figs. 3 and $4 \mathrm{~A})$; ii) incidence of the cancer spreading in the liver and lung was significantly decreased using Fisher's exact analysis (Table I); iii) number of liver cancer spreading nodules was significantly decreased using paired t-test $(\mathrm{P}<0.001$; Fig. $4 \mathrm{~B})$; and iv) there was a marked decrease in the number of the leukemia cells in lung, liver and spleen on H\&E staining sections (Fig. 5A-D). These data provide strong evidence that the CD176 antibody treatment effectively inhibits the growth and spreading of $\mathrm{CD}_{176^{+}}$ leukemia cells.

Number of leukemia cells in bone marrow is decreased by the CD176 antiserum treatment alone. Through histopathological observation on bone marrow smears with Giemsa staining and subsequent statistical analysis, the CD176 antiserum treatment alone significantly reduced the percentage of leukemia cells in total bone marrow cells (Fig. 5E-H, Table II). The CD176 antibody treatment also inhibited the growth of $\mathrm{CD}_{176}{ }^{+}$leukemia cells in bone marrow.

\section{Discussion}

Immunotherapy is of considerable benefit to cancer patients (20). Anticancer vaccines targeting tumor-associated carbohydrates, such as CD176, provide an attractive approach for the prevention and treatment of cancer. Several studies suggested that the vaccination with a CD176 antigen could effectively improve breast cancer patient survival $(5,14,15)$. In patients immunized with CD176 antigen, aside from the activation of CD176-specific antibody responses, a CD176-specific DTH reflecting the activation of specific $\mathrm{T}$ cells was also observed (5). An animal study also showed that mice immunized with synthetic CD176 glycopeptide vaccines generated a cytotoxic $\mathrm{T}$ cell response to $\mathrm{CD}_{176^{+}}$tumor cells (21). These results indicated that tumor vaccines addressing CD176 (TF antigen) could activate a specific humoral and cellular antitumor response. In the present study, we used $\mathrm{CD} 176^{+}$leukemia mice as experimental models and titers of the CD176 IgM and $\mathrm{IgG}$ antibody as immune response index. We found that the mice pre-immunized with CD176 antigen had a significant survival advantage compared to the control mice without the immunization. These observations indicated that high anti-CD176 immune responses and high titers of CD176 antibodies may be beneficial for the prevention of tumor development. Natural antibodies against CD176 occurring in all adult sera (22) may be part of a mechanism of humoral immunosurveillance against 

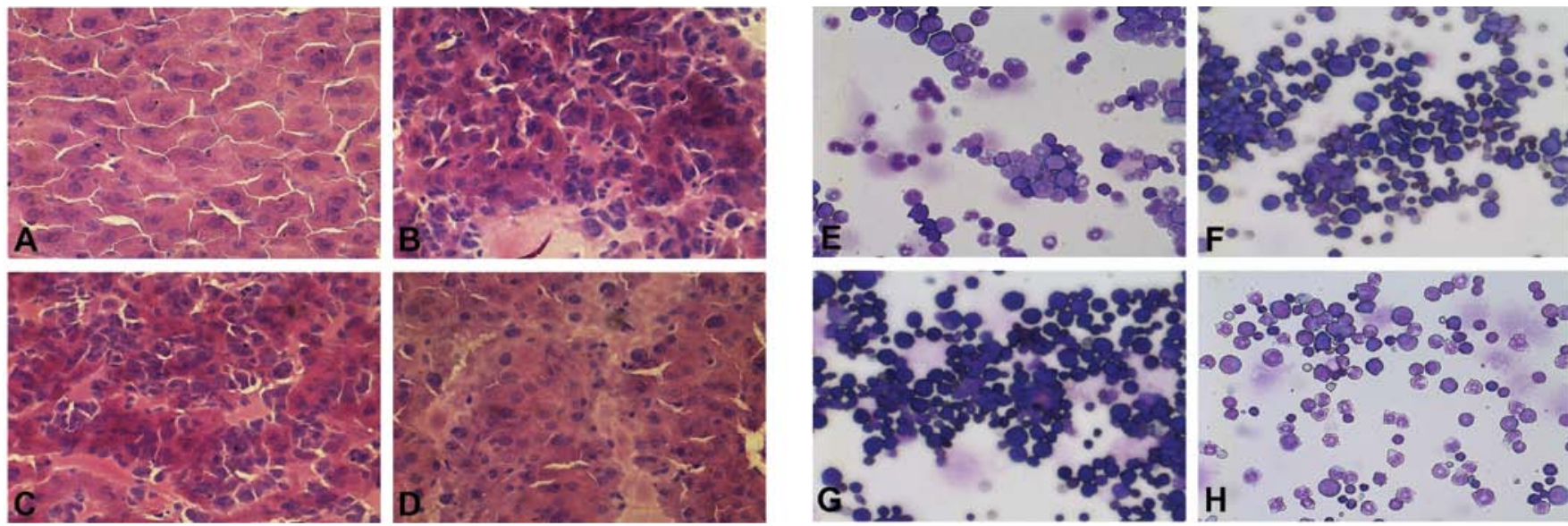

Figure 5. Histopathological analysis shows that the CD176 antiserum treatment inhibits the growth and metastasis of CD176 cancer cells in liver (A-D) and bone marrow (E-H). (A) Liver section of the normal mouse; (B) liver section of the CD176 leukemia mouse; (C) liver section of the CD176 ${ }^{+}$leukemia mouse treated with the normal mouse serum; (D) liver section of CD176 leukemia mouse treated with the CD176 antiserum. Note that the leukemic cells were reduced by the CD176 antiserum treatment (magnification, $\mathrm{x} 400)$. (E) Bone marrow smear of the normal mouse; $(\mathrm{F})$ a bone marrow smear of the $\mathrm{CD} 176^{+}$ leukemia mouse; $(\mathrm{G})$ bone marrow smear of the $\mathrm{CD}_{176^{+}}$leukemia mouse treated with the normal mouse serum; $(\mathrm{H})$ bone marrow smear of the $\mathrm{CD} 176^{+}$ leukemia mouse treated with the CD176 antiserum. Note that CD176 antiserum treatment reduced the percentage of the leukemic cells in comparison to the total bone marrow cells (magnification, $\mathrm{x} 400$ ).

CD176 ${ }^{+}$tumor cells (13), but the natural CD176 antibody is likely to be consumed and is present in lower titer in cancer patients. Thus, we presented a hypothesis that the vaccination with CD176 antigen increased and further maintained the high anti-CD176 immune response and high CD176 antibody titers for the prevention of tumor recurrence and metastasis. The development of new tumor vaccines addressing CD176 merits further consideration.

In clinical medicine, antibody treatment has unique advantages for cancer patients. For advanced cancer patients and immunocompromised patients, passive antibody immunotherapy is better than tumor vaccine immunotherapy. Antibody drugs have been widely used for cancer treatment including leukemia. For example, trastuzumab which triggers apoptosis of HER2-positive breast cancer (16), and rituximab which targets surface antigen CD20 of B-cell malignancies, have been used for clinical treatment (23). In previous studies, we have found that CD176 antibody can induce apoptosis of CD176+ leukemia cells $(13,17)$. Several studies have shown that $\mathrm{CD}_{176}{ }^{+}\left(\mathrm{TF}^{+}\right) \mathrm{T}$-cell acute lymphoblastic leukemia (ALL) has a better prognosis than CD176- $\operatorname{ALL}(7,8)$. Apoptotic destruction induced by CD176 antibody of the natural antibody repertoire may provide an explanation for this phenomenon (13). Tumor-specific apoptosis mediated by natural carbohydrate-reactive $\operatorname{IgM}$ antibodies in humans has been observed and may lead to new strategies for anticancer immunotherapy (24). Additionally, CD176 antibody treatment can prevent tumor metastasis (18). Based on these findings, the application of the CD176 antibody as an anticancer drug may be a promising approach for current cancer immunotherapy. In order to investigate whether the CD176 antibody treatment alone has a therapeutic effect on animals with $\mathrm{CD} 176^{+}$cancer cells in vivo, we performed passive transfer of the CD176 antiserum in CD176 ${ }^{+}$leukemia mice. Several inhibitory experiments with TF (CD176) glycan demonstrated that the CD176 antiserum used in the study reacted only with the tumor-associated CD176 in cancer cells. The present study clearly demonstrated that passive immunotherapy using the CD176 antiserum alone provided $\mathrm{CD} 176^{+}$leukemia mice with a significant advantage for survival. Furthermore, we observed that the CD176 antiserum treatment alone could inhibit the growth and spreading of $\mathrm{CD}_{176^{+}}$cancer cells in bone marrow, spleen, liver and lung as determined by histopathological examination. Although the mechanisms of the CD176 antibody treatment were not fully understood, CD176 antibody treatment may be involved in the following process: i) CD176 antibody could inhibit $\mathrm{CD}_{176}{ }^{+}$cancer cell metastasis to bone marrow, spleen, lung and liver through blocking the adhesion of cancer cells to the endothelium $(11,25)$ and hepatocytes (10); ii) CD176 antibody could mediate the elimination of $\mathrm{CD} 176^{+}$cancer cells through complement-dependent cytotoxicity (CDC) and/or antibody-dependent cellular cytotoxicity (ADCC) performed by natural killer cells, neutrophils, and macrophages; and finally iii) CD176 antibody could induce apoptosis of CD176 leukemia cells $(13,17)$.

In conclusion, the present study provided strong evidence that both CD176 antigen-based active-immunotherapy and CD176 antibody-based passive-immunotherapy lead to a therapeutic response in $\mathrm{CD}_{176^{+}}$leukemia mice in vivo. Since CD176 is expressed on the surface of human leukemic cells but is almost absent in normal and benign adult human tissues (3), CD176 may be an ideal target for anti-leukemia immunotherapy. Therefore, both CD176 vaccine and CD176 antibody drug may be beneficial to $\mathrm{CD} 176^{+}$leukemia patients. The modes of application could be selected according to the clinical situations of the respective leukemia.

\section{Acknowledgements}

The present study was financially supported by grants from the National Natural Science Foundation of China (no. 81072563), the Yunnan Province Science and Technology Department (2008CCO15, 2011CI139) and the Chinese Academy of Sciences (KSCX2-YW-R-196). 


\section{References}

1. Goletz S, Cao Y, Danielcyk A, Ravn P, Schoeber U and Karsten U: Thomsen-Friedenreich antigen: the 'hidden' tumour antigen. Adv Exp Med Biol 535: 147-162, 2003.

2. Cao Y, Merling A, Karsten U and Schwartz-Albiez R: Expression of Thomsen-Friedenreich-related carbohydrate antigens on human leukemia cells. In: Leucocyte Typing VII. Mason D, et al. (eds). Oxford University Press, Oxford, pp204-205, 2002.

3. Cao Y, Stosiek P, Springer GF and Karsten U: ThomsenFriedenreich-related carbohydrate antigens in normal adult human tissues: a systematic and comparative study. Histochem Cell Biol 106: 197-207, 1996.

4. Springer GF: T and Tn, general carcinoma autoantigens. Science 224: 1198-1206, 1984.

5. Springer GF: Immunoreactive $\mathrm{T}$ and $\mathrm{Tn}$ epitopes in cancer diagnosis, prognosis, and immunotherapy. J Mol Med 75: 594-602, 1997.

6. Aller CT, Kucuk O, Springer GF and Gilman-Sachs A: Flow cytometric analysis of $\mathrm{T}$ and $\mathrm{Tn}$ epitopes on chronic lymphocytic leukemia cells. Am J Hematol 52: 29-38, 1996.

7. Kaspers GJ, Veerman AJ, Van Wering ER, et al: Prognostic significance of peanut agglutinin binding in childhood acute lymphoblastic leukemia. Leukemia 10: 675-681, 1996.

8. Veerman AJ, Hogeman PH, Huismans DR, Van Zantwijk CH and Bezemer PD: Peanut agglutinin, a marker for T-cell acute lymphoblastic leukemia with a good prognosis. Cancer Res 45: 1890-1893, 1985.

9. Lin WM, Karsten U, Goletz S, Cheng RC and Cao Y: Expression of CD176 (Thomsen-Friedenreich antigen) on lung, breast and liver cancer-initiating cells. Int J Exp Pathol 92: 97-105, 2011

10. Cao Y, Karsten UR, Liebrich W, Haensch W, Springer GF and Schlag PM: Expression of Thomsen-Friedenreich-related antigens in primary and metastatic colorectal carcinomas. A reevaluation. Cancer 76: 1700-1708, 1995.

11. Glinsky VV, Glinsky GV, Rittenhouse-Olson $\mathrm{K}$, et al: The role of Thomsen-Friedenreich antigen in adhesion of human breast and prostate cancer cells to the endothelium. Cancer Res 61 : 4851-4857, 2001.

12. Heimburg J, Yan J, Morey S, et al: Inhibition of spontaneous breast cancer metastasis by anti-Thomsen-Friedenreich antigen monoclonal antibody JAA-F11. Neoplasia 8: 939-948, 2006.
13. Cao Y, Merling A, Karsten U, et al: Expression of CD175 (Tn), CD175s (sialosyl-Tn) and CD176 (Thomsen-Friedenreich antigen) on malignant human hematopoietic cells. Int J Cancer 123: 89-99, 2008.

14. MacLean GD, Bowen-Yacyshyn MB, Samuel J, et al: Active immunization of human ovarian cancer patients against a common carcinoma (Thomsen-Friedenreich) determinant using a synthetic carbohydrate antigen. J Immunother 11: 292-305, 1992.

15. Slovin SF, Ragupathi G, Musselli C, et al: Thomsen-Friedenreich (TF) antigen as a target for prostate cancer vaccine: clinical trial results with TF cluster (c)-KLH plus QS21 conjugate vaccine in patients with biochemically relapsed prostate cancer. Cancer Immunol Immunother 54: 694-702, 2005.

16. Dougan M and Dranoff G: Immune therapy for cancer. Annu Rev Immunol 27: 83-117, 2009.

17. Yi B, Zhang M, Schwartz-Albiez R and Cao Y: Mechanisms of the apoptosis induced by CD176 antibody in human leukemic cells. Int J Oncol 38: 1565-1573, 2011.

18. Shigeoka H, Karsten U, Okuno K and Yasutomi M: Inhibition of liver metastases from neuraminidase-treated colon 26 cells by an anti-Thomsen-Friedenreich-specific monoclonal antibody. Tumor Biol 20: 139-146, 1999.

19. Karsten U, Butschak G, Cao Y, Goletz S and Hanisch FG: A new monoclonal antibody (A78-G/A7) to the Thomsen-Friedenreich pan-tumor antigen. Hybridoma 14: 37-44, 1995.

20. Topalian SL, Weiner GJ and Pardoll DM: Cancer immunotherapy comes of age. J Clin Oncol 29: 4828-4836, 2012.

21. Xu Y, Gendler SJ and Franco A: Designer glycopeptides for cytotoxic T cell-based elimination of carcinomas. J Exp Med 199: 707-716, 2004.

22. Butschak $\mathrm{G}$ and Karsten U: Isolation and characterization of thomsen-friedenreich-specific antibodies from human serum. Tumor Biol 23: 113-122, 2002.

23. Maloney DG: Anti-CD20 antibody therapy for B-cell lymphomas. N Engl J Med 366: 2008-2016, 2012.

24. Schwartz-Albiez R: Naturally occurring antibodies directed against carbohydrate tumor antigens. Adv Exp Med Biol 750: 27-43, 2012.

25. Glinskii OV, Sud S, Mossine VV, et al: Inhibition of prostate cancer bone metastasis by synthetic TF antigen mimic/galectin-3 inhibitor lactulose-L-leucine. Neoplasia 14: 65-73, 2012. 